SOI: $1.1 /$ TAS DOI: $10.15863 /$ TAS

International Scientific Journal Theoretical \& Applied Science

p-ISSN: 2308-4944 (print) $\quad$ e-ISSN: 2409-0085 (online)

Year: $2015 \quad$ Issue: $09 \quad$ Volume: 29

Published: $30.09 .2015 \quad \underline{\text { http://T-Science.org }}$
Nikolai Vladimirovich Semenchenko

Student of the Department «Equipment and technology of machine-building production» FSBEI HPE «Togliatti State University», Russia bonder64@mail.ru

Konstantin Olegovich Hryachkov Student of the Department «Equipment and technology of machine-building production»» FSBEI HPE «Togliatti State University», Russia

SECTION 7. Mechanics and machine construction.

\title{
MATHEMATICAL MODELS FOR CALCULATING GEOMETRICAL AND FORCE PARAMETERS OF DEFORMATION CLADDING PROCESS
}

Abstract: This article describes the main mathematical models describing the deformation cladding by flexible tool; describes changes that happened with the mathematical models. We have defined the model that best describes the process. Possible direction of further development was revealed.

Key words: flexible tool; cladding; coating method; mathematical model.

Language: Russian English

Citation: Semenchenko NV, Hryachkov KO (2015) MATHEMATICAL MODELS FOR CALCULATING GEOMETRICAL AND FORCE PARAMETERS OF DEFORMATION CLADDING PROCESS. ISJ Theoretical \& Applied Science 09 (29): 115-118.

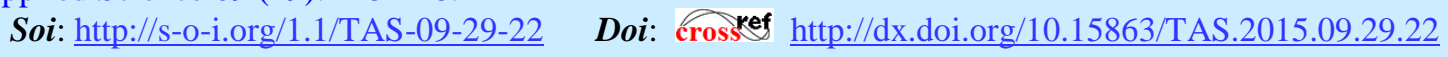

\section{МАТЕМАТИЧЕСКИЕ МОДЕЛИ РАСЧЕТА ГЕОМЕТРИЧЕСКИХ И СИЛОВЫХ ПАРАМЕТРОВ ПРОЦЕССА ДПГИ}

Аннотация: В данной статье рассмотрены основные математические модели, описывающие метод деформационного плакирования гибким инструментом, рассмотрены изменения, произошедшие с данными моделями. Определена модель, наиболее точно описывающая процесс ДПГИ. Выявлено возможное направление дальнейшего развития данного метода нанесения покрытий.

Ключевые слова: гибкий инструмент; плакирование; метод нанесения покрытий; математическая модель.

Деформационное плакирование гибким инструментом (ДПГИ) - один из универсальных методов модификации поверхностного слоя деталей пар трения, позволяющий наносить покрытие с одновременной упрочняющей обработкой [1]. Данный метод имеет богатую историю. Изобретателями было предложено множество возможных схем реализации процесса, различные методы интенсификации процесса дополнительными физическими воздействиями, проработаны конструкции гибкого инструмента, элемента из материала покрытия и конструкций его подачи в зону обработки. Что позволило использовать данный метод для различного сочетания условий процесса, с различными материалами детали, инструмента и материала покрытия.

Дальнейшее развитие данного метода предполагает исследования направленные на автоматизацию процесса. Что позволит стабилизировать основные энергосиловые параметры процесса (скорости вращения ворса гибкого инструмента, усилия прижатия механической щетки к обрабатываемому изделию, температуры процесса и д.р.). И как следствие, позволит протекать процессу в установившемся режиме, поддерживая физикомеханические характеристики формируемых покрытий на высоком уровне. Автоматизация процесса предусматривает управление основными параметрами, следовательно, необходимы зависимости этих параметров от входных параметров процесса плакирования. Что ставит пред нами задачу детального изучения математических моделей описывающих данный процесс.

Различные подходы к разработке моделей расчета параметров процесса ДПГИ в различных работах определяются их авторами в зависимости от исходных предположений о характере 
взаимодействия гибких элементов проволочного инструмента с обрабатываемой поверхностью изделия и допущениями, которые заложены ими в расчетную схему (рис. 1).

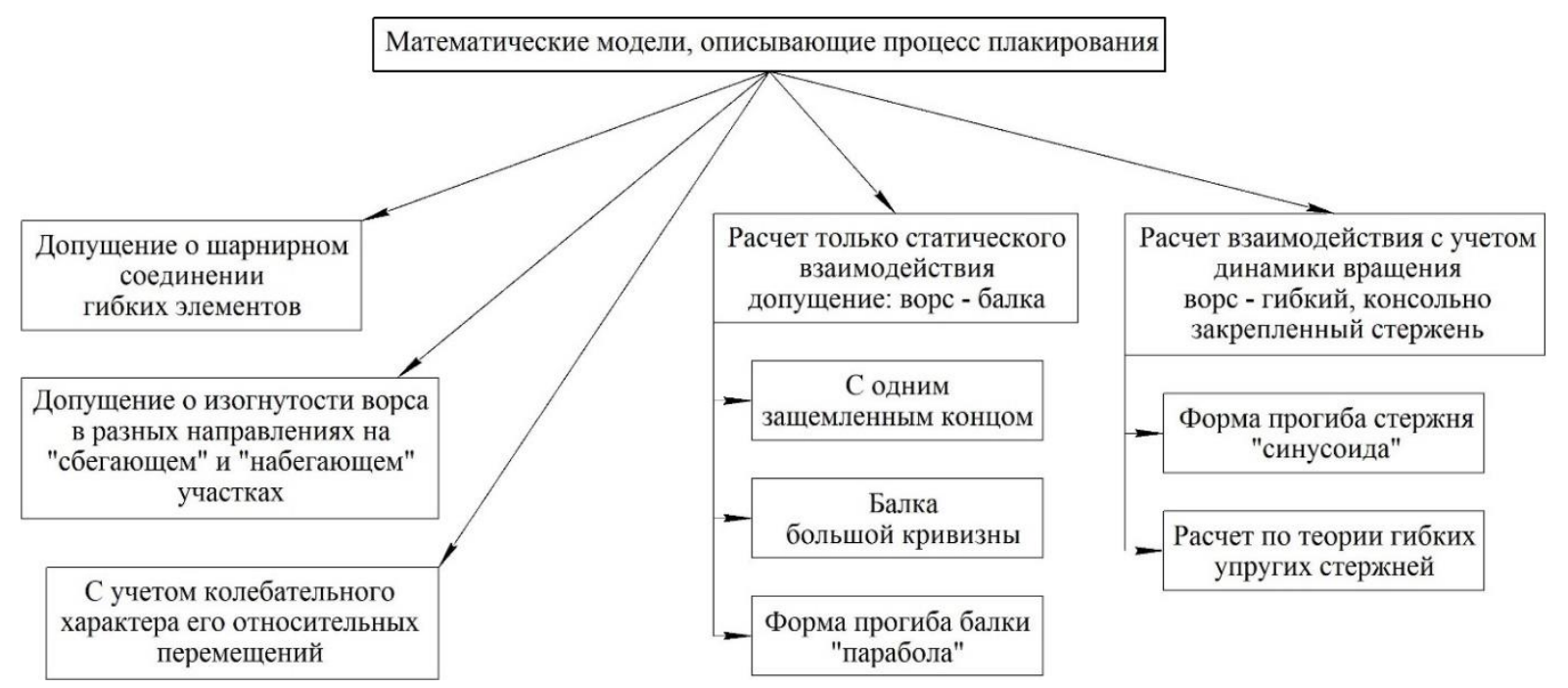

Рисунок 1 - Классификация математических моделей расчета основных параметров процесса ДПГИ.

Так, например, авторы методики [2] Проскуряков Ю.Г., Ершов В.С. сделали допущение, что ворс механической щетки закреплен шарнирно, в то время как для процесса плакирования используется гибкий инструмент с жесткой заделкой проволочных элементов. Что делает невозможным использование данной методики для расчета параметров процесса плакирования.

Автор работы [3] Ершов В.С. предполагает, при расчете геометрических параметров процесса, что ворс щетки на «набегающем» и «сбегающем» участках зоны контакта изогнут в противоположные стороны, как было бы при статическом прижатии гибких элементов к обрабатываемой поверхности. Данная модель так же не подходит для определения параметров процесса плакирования, так как в реальности ворс гибкого инструмента изгибается во время вращения щетки только в одну, противоположную вращению, сторону.

Исследователи в работах [4-6] рассматривают ворс механической щетки как упругую балку при статическом взаимодействии с поверхностью. Авторы представляют ворс, как балку с одним жестко защемленным концом, к которой приложена статическая нагрузка. В работе [7] гибкий элемент (ворс щетки) рассматривается как параболически изогнутая балка, имеющая жесткое закрепление на одном конце. В данном случае математическая модель основана на решении уравнения изогнутой оси металлического ворса в виде параболы. Похожий подход использовался и в работе [18], в которой изогнутые гибкие элементы, находящиеся в контакте с обрабатываемой поверхностью, рассматриваются в виде балок с большими прогибами. В рассмотренных моделях процесса обработки щетками никак не учтен ударных характер взаимодействия гибких элементов проволочного инструмента с обрабатываемой поверхностью детали. Также, не учтено увеличение силы взаимодействия ворса щетки с поверхностью детали за счет кинетической энергии вращения инструмента. Хотя давления, возникаемые в зоне обработки, будут значительно больше при динамическом воздействии, чем при статическом.

В работах [8, 9] Кургузовым Ю.И. была разработана методика, учитывающая больше аспектов процесса деформационного плакирования, чем в рассмотренных ранее работах. В них форма изгибающейся части металлического ворса определена синусоидой, что было получено на основе экспериментальных данных; в ней учтен так же характер ударной обработки. Эта методика успешно развита в работе [1] Анцуповым В.П., в которой предложены математические модели, описывающие процесс плакирования для вогнутых цилиндрических и плоских поверхностей. Методики $[1,8,9]$ определения геометрических и силовых параметров гораздо точнее своих предшественников, но они и дальше продолжали свое развитие. В работе [17] автором рассмотрено взаимодействие гибкого ворса с поверхностью детали и ЭМП не как сухое трение твердых тел, в связи с чем, было получено более 
полное описание процесса взаимодействия инструмента с деталью.

Автором [10], Зотовым А.В., представлена математическая модель расчета геометрических и силовых параметров зоны плакирования, построенная посредством метода эллиптических параметров [11], основанного на точном решении дифференциального уравнения упругой линии (теория гибких упругих стержней), что позволило получить еще более точные результаты. Данная модель позволяет рассчитать с высокой точностью не только силы взаимодействия, вращающие моменты и величину прогибов ворса, но также и напряжения, возникающие в ворсе.

Одной из самых новых работ является [12], где автором Леванцевичем М.А. разработана, отличающаяся от остальных, математическая модель, описывающая процесс плакирования с точки зрения сплошности получаемого при обработке покрытия. Данная модель описывает колебательный характер относительных перемещений металлического ворса проволочного инструмента с момента его входа в зону контакта с обрабатываемой деталью и на протяжении всего времени контактирования. Максимченко Н.Н. проверена созданная им методика расчета параметров процесса плакирования для получения необходимой сплошности наносимого покрытия [13].

Проанализированные математические модели достаточно подробно отображают процесс развития методик расчета параметров плакирования. От менее точных, неприменимых для определенных условий, моделей до более точных, позволяющих с высокой достоверностью определять параметры технологического процесса. В процессе развития математические модели претерпевали изменения, постоянно наращивая сложность расчетов, учитывая все больше и больше различных факторов, влияющих на процесс. Что позволило добиться серьезного повышения соответствия расчетных и реальных значений величин, характеризующих процесс плакирования.

Наибольший интерес представляет математическая модель [10], позволяющая определять геометрические и энергосиловые параметры зоны контакта с точность до 2-6\% [14]. По данной методике был разработан алгоритм расчета геометрических и силовых параметров зоны контакта, представляющий собой набор циклов с последовательным повышением точности вычисления. Данный алгоритм позволяет рассчитать параметры процесса, затрачивая на процедуру минимум времени [15]. Полученная точность модели является достаточной для разработки на ее основе системы управления процессом плакирования. Разработка такой системы может заключаться в развитии модели адаптивной системы управления представленной в работе [16].

Установив такую систему, появляется возможность компенсации динамических и температурных погрешностей обработки, поддержания необходимой контактной силы, и как следствие повышение качества обработки.

Исследование выполнено под руководством Гуляева В.А., к.т.н, доцент и Зотова А.В., cm. преп. кафедры «Оборудование $и$ технологии машиностроительного производства» ФГОБУ ВПО «Тольяттинский государственный университет», Россия.

\section{References:}

1. Ancupov VP (1999) Teorija i praktika plakirovanija izdelij gibkim instrumentom. Magnitogorsk: MGTU im. G.I. Nosova, 1999. $241 \mathrm{p}$.

2. Proskurjakov JG, Ershov VS (1970) Issledovanie zony kontakta mehanicheskoj shhetki s obrabatyvaemym izdeliem // Issledovanie tehnologicheskih processov uprochnjajushhe-kalibrujushhej i formoobrazujushhej obrabotki metallov: Mezhvuz. sb. Rostov-na-Donu. 1970. pp. 144154.

3. Ershov VS (1969) Geometrija processa chistovoj obrabotki detalej mehanicheskimi shhetkami // Tehnologija proizvodstva sel'skohozjajstvennyh mashin: Mezhvuz. sb. Rostov-na-Donu. 1969. pp. 109-118.

4. Makarov AN, Belevskij LS, Kadoshnikov VI (1985) Opredelenie jenergosilovyh parametrov pri obrabotke metallicheskimi shhetkami // Teorija i praktika proizvodstva metizov: Mezhvuz. sb. Sverdlovsk. 1985. Vyp. 12. pp. 29-32.

5. Belevskij LS, Kadoshnikov VI, Melent'eva EJ (1988) Opredelenie jenergosilovyh parametrov pri nanesenii metallicheskih pokrytij mehanicheskim sposobom // Teorija i praktika proizvodstva metizov: Mezhvuz. sb. Sverdlovsk. 1988. Vyp. 14. pp. 98-104. 


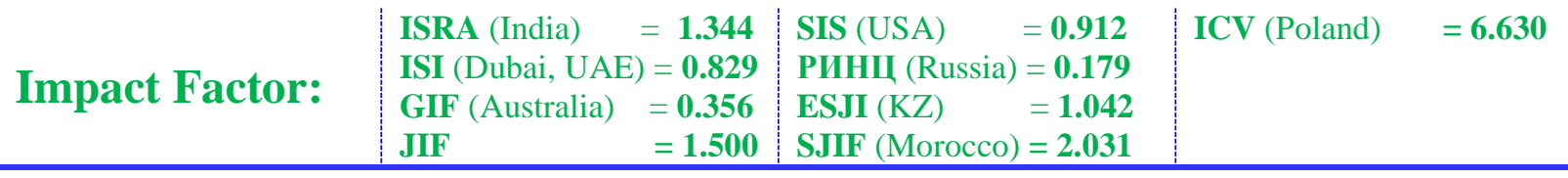

6. Kadoshnikov VI (1988) Sovershenstvovanie tehnologii proizvodstva bimetallicheskoj stalealjuminevoj provoloki primeneniem novogo sposoba nanesenija promezhutochnogo sloja: Dis. kand. tehn. nauk. Magnitogorsk, 1988. $192 \mathrm{p}$.

7. Belevskij LS, Sankin JV (1989) Analiz geometricheskih i jenergiticheskih parametrov zony kontakta vorsa metallicheskoj shhetki s obrabatyvaemoj poverhnost'ju // Teorija i praktika proizvodstva metizov: Mezhvuz. sb. Magnitogorsk: MGMI, 1989. Vyp. 15. pp. 169178.

8. Kurguzov JI, Papshev DD (1986) Tehnologicheskoe obespechenie kachestva poverhnosti pri uprochnenii mehanicheskimi shhetkami // Vestnik mashinostroenija. 1986. № 4. pp. 54-58.

9. Kurguzov JI (1981) Tehnologicheskoe obespechenie kachestva poverhnostnogo sloja zakalennyh detalej obrabotkoj mehanicheskimi shhetkami: Dis. kand. tehn. nauk. - Kujbyshev, 1981. - $235 \mathrm{p}$.

10. Zotov AV (2012) Metodika rascheta geometricheskih i silovyh parametrov zony kontakta pri plakirovanii izdelij gibkim instrumentom // Vektor nauki Tol'jattinskogo gosudarstvennogo universiteta. 2012. № 3. pp. 85-88.

11. Popov EP (1986) Teorija gibkih uprugih sterzhnej. M.: Nauka, 1986. 296 p.

12. Levancevich MA (1987) Model' kontaktnogo vzaimodejstvija vrashhajushhejsja metallicheskoj shhetki s poverhnost'ju pri deformacionnom plakirovanii: Mezhvuz. sb. nauchn. tr. Sverdlovsk: UPI, 1987. Vyp. 11. pp. 24-27.

13. Maksimchenko NN (2009) Tehnologicheskoe obespechenie jekspluatacionnyh harakteristik napravljajushhih skol'zhenija formirovaniem antifrikcionnyh pokrytij gibkim instrumentom: Dis. kand. tehn. nauk. - Minsk, 2009. - 131 p.

14. Zotov AV, Drachev OI, Semenchenko NV (2013) Analiz naprjazhennogo deformirovannogo sostojanija provolochnyh jelementov gibkogo instrumenta // Izvestija Volgogradskogo gosudarstvennogo tehnicheskogo universiteta: mezhvuz. cb. nauch. st. №20 (123) / VolgGTU. - Volgograd, 2013. - $124 \mathrm{p}$

15. (2014) Svidetel'stvo o gosudarstvennoj registracii programmy dlja JeVM № 2014612918. Rossijskaja Federacija. Raschet parametrov ploskogo kontakta pri plakirovanii provolochnym instrumentom / Bobrovskij A.V., Zotov A.V., Semenchenko N.V.; zajavitel' i pravoobladatel' FGBOU VPO «Tol'jattinskij gosudarstvennyj universitet». - № 2014610212; zajavl. 16.01.2014; opubl. 20.04.2014, Reestr programm dlja JeVM - 1 p.

16. Semenchenko NV, Guljaev VA, Sevost'janov AS (2014) Avtomatizacija processa plakirovanija gibkim instrumentom ploskih poverhnostej detalej // Akademicheskaja nauka - problemy i dostizhenija n.-i. c. «Akademicheskij». - Tom. 2. / North Charleston, SC, USA, 2014. - pp. 127-130.

17. Onshin NV (2001) Razrabotka i issledovanie metoda drobnogo plakirovanija gibkim instrumentom dlja proizvodstva bimetallicheskih detalej metallurgicheskogo oborudovanija povyshennoj iznosostojkosti: Dis. kand. tehn. nauk. Magnitogorsk, 2001. 119 p.

18. Perepichka EV (1989) Ochistnouprochnjajushhaja obrabotka izdelij shhetkami - M.: Mashinostroenie, 1989. - 136 p. 